\title{
Measurements of human motor and visual activities with diffusing-wave spectroscopy
}

\author{
J. Li, G. Dietsche, G. Maret and T. Gisler \\ Universität Konstanz, Fachbereich Physik, 78457 Konstanz, Germany \\ thomas.gisler@uni-konstanz.de \\ B. Rockstroh and T. Elbert \\ Universität Konstanz, Fachbereich Psychologie, 78457 Konstanz, Germany \\ S. E. Skipetrov \\ Laboratoire de Physique et Modélisation des Milieux Condensés/CNRS, Université Joseph Fourier, 38042 Grenoble, France
}

\begin{abstract}
Diffusion coefficients in the human sensorimotor and visual cortices were measured using diffusing-wave spectroscopy. Motor and visual activation leads to increases of the diffusion coefficients in the respective cortical areas over the values at rest.
\end{abstract}

OCIS codes: (030.5260) Photon counting; (030.6140) Speckle; (170.3660) Light propagation in tissues; (170.7050) Turbid media; (290.7050) Turbid media

\begin{abstract}
1. Introduction
Near-infrared light has become an increasingly important tool for the non-invasive investigation of brain functional activity [1-6]. Neuronal and hemodynamic activities during brain activation are accompanied by changes in the cortical optical parameters that can be measured by continuous-wave, time-domain and frequency-domain methods [4]. Diffusing-wave spectroscopy (DWS) [7-8] has the ability to detect dynamical heterogeneities inside turbid media even without static (scattering or absorption) contrast $[9,10]$, which provides a new way to measure functionrelated hemodynamics such as the enhanced cerebral blood flow during brain activation [11,12]. Using DWS it could also be possible to measure neuronal vesicle motion that is more directly related to the neuron activity. In this study, we use DWS to investigate, in an entirely non-invasive way, the responses of the human brain to motor and visual stimulation. An analytical solution of the correlation-diffusion equation with a 3-layer head model (modelling the dynamics in the cortical tissue by Brownian motion) [13] is used to determine the effective diffusion coefficient within $\mathrm{cm}$-sized areas of the sensorimotor and visual cortices from the measured autocorrelation functions. Stimulation of the sensorimotor and visual cortices by motor tasks and flickering, respectively, is found to lead to significant and specific increases in the diffusion coefficients in the respective cortical areas.
\end{abstract}

\section{Method}

A. Diffusing-wave spectroscopy and experimental setup

For the DWS experiment we use near-infrared light from a diode laser operating in a single longitudinal mode at $802 \mathrm{~nm}$ (TA-100, Toptica) which is guided to the subject's scalp via a multi-mode fiber. A few-mode fiber (FS-SN6324, Thorlabs) transmitting 6 transverse modes at $802 \mathrm{~nm}$ is used to pick up the diffusely transmitted light at a distance from the source. The light is detected by a single-photon counting module (SPCM-AQR-15-FC, PerkinElmer), and the autocorrelation function $g_{2}(\tau)=\langle I(t) \cdot I(t+\tau)\rangle /\langle I(t)\rangle^{2}$ of the measured intensity $I(t)$ is then computed by a digital correlator (ALV-5000E, ALV). In our data analysis, the effective cortical diffusion coefficient was estimated by fitting the measured autocorrelation functions to an analytical solution of the correlation-diffusion equation for a 3-layer head model representing scalp, skull and brain, modelling the dynamics within the cortical tissue by Brownian motion [13].

\section{B. Protocol}

For the motor experiments, a source-receiver pair separated by $20-25 \mathrm{~mm}$ was placed over the primary sensorimotor cortex (C3 in the international 10-20 system) of 11 right-handed subjects ( 3 male, 8 female). Self-paced finger opposition was used to stimulate the motor cortex. One measurement block consisted of 100s stimulation periods followed by 100s periods of rest. For the visual experiments, the source-receiver pair was fixed over the primary visual cortex (around O2) of 2 female subjects. $5 \mathrm{~Hz}$ flickering of a computer screen was used as visual stimulation. 
A measurement block consisted of 40s of stimulation and 40s periods of rest during which the subjects closed their eyes. During the data collection, the correlator was set to work in multiple-run mode, each run lasting 10s.

\section{Results}

Fig. 1 shows the change of the diffusion coefficient of the sensorimotor cortex during the finger opposition task with the right (contralateral) and with the left (ipsilateral) hand relative to the respective resting baseline values. Both contra- and ipsilateral stimulations show enhanced cortical dynamics. We find that the contralateral response (approximately 39\%) is stronger than the ipsilateral (20\%) [13]

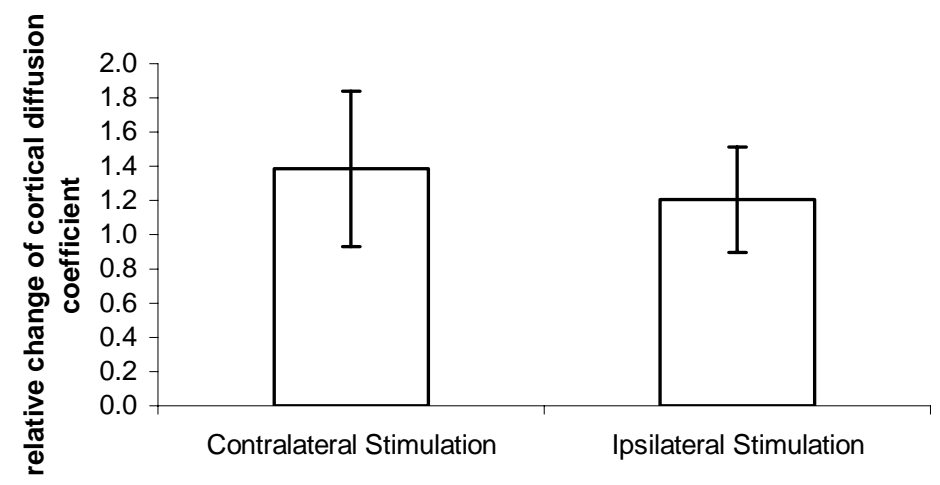

Fig. 1: Relative change of the diffusion coefficient of the sensorimotor cortex upon motor stimulation. Error bars are standard deviations calculated from 11 subjects.

Fig. 2 shows the response of the diffusion coefficient of the visual cortex upon stimulation by $5 \mathrm{~Hz}$ flickering for 2 subjects. The relative change of cortical diffusion coefficient is approximately $20 \%$ during stimulation periods.

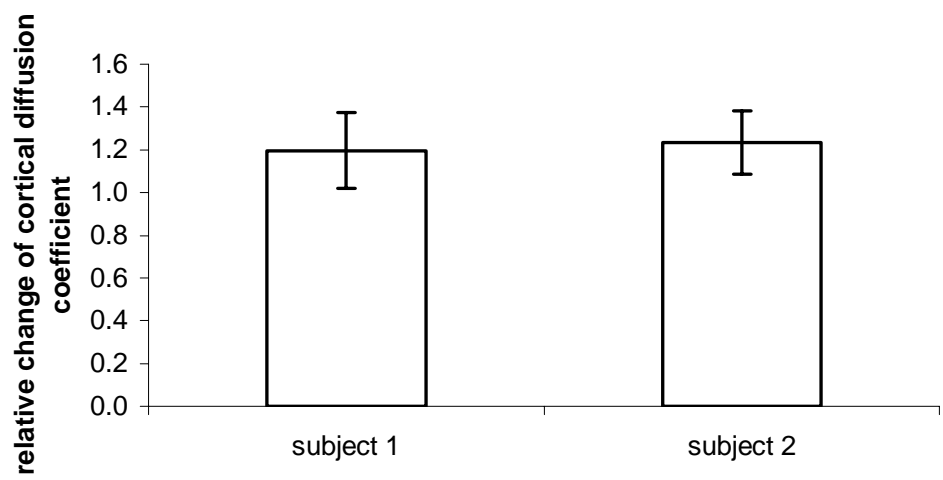

Fig. 2: Relative change of the diffusion coefficient of the visual cortex measured from 2 subjects upon $5 \mathrm{~Hz}$ flickering stimulation. Error bars for each subject are standard deviations obtained from 4 measurements.

\section{Discussion}

While brain functional activation is accompanied by a regional enhancements of blood flow, neuronal vesicle mobility and axonal swelling, it is not clear at present which one of these effects dominates the functional enhancement of the effective cortical diffusion coefficient measured by DWS. An answer to this question might come from a comparison of DWS data with blood flow rates measured simultaneously with PET or fMRI, which requires a reliable estimation of cortical diffusion coefficients from DWS data. So far, however, non-invasive functional DWS data from the brain have been analyzed in terms of a semi-infinite 1-layer model [11,12]. Our numerical simulations show that with this simple head model, the cortical diffusion coefficient is not only underestimated (by as much as a factor of 10) but also biased inconsistently depending on the underlying cortical dynamics. Therefore even relative changes of cortical dynamics cannot be obtained accurately from the semi-infinite 1-layer head model. In our data analysis, we modelled the head as a 3-layer medium representing the scalp, skull and brain. Although several studies have shown that the effect of cerebral spinal fluid (CSF) is important for conventional optical methods based on optical contrast, our Monte Carlo simulation shows that the effect of this thin 
and clear layer on the autocorrelation function is only minor, resulting in a slightly underestimated diffusion coefficient. Thus there is no necessity to include this layer in the head model for interpreting brain DWS data. The SNR of the correlation function strongly depends on the measured photon count rate especially at the short time lags which contain most of the information on the dynamics of deeper layers. In order to achieve a large count rate and meanwhile keep the safe limit of $4 \mathrm{~mW} / \mathrm{mm}^{2}$, we used a circular source with a diameter of $4 \mathrm{~mm}$. Theoretical analysis shows that the correlation function of this extended source is only slightly different from that of the point source in the case of large source-detector spacing (e.g., $>20 \mathrm{~mm}$ ).

Our data analysis shows that describing the tissue dynamics by Brownian diffusion yields a better agreement between theory and data than a random flow model. This observation is consistent with the result of Cheung et al. [11]. Our motor results show that the cortical dynamics increases by $39 \%$ for the contralateral stimulation [13]. This change is in the same range (29\%-49\%) as observed by Durduran [11]. For our visual experiments, upon $5 \mathrm{~Hz}$ flickering stimulation the dynamics in the primary visual cortex increases by $20 \%$. This result is within the range of PET measurements of the cerebral blood flow changes $(16 \pm 16) \%$ for $2 \mathrm{~Hz}$ and $(68 \pm 20) \%$ for $8 \mathrm{~Hz}$ flickering stimulation [14].

Our results suggest that the microscopic motions within cortical tissue upon which DWS is based might provide a unique diagnostic modality for non-invasive brain investigation.

\section{References}

1. F.F. Jöbsis, Science 198, 1264 (1977).

2. A. Yodh and B. Chance, Physics Today 48, 34 (1995).

3. M. A. Franceschini, V. Toronov, M.E. Filiaci, E. Gratton, and S. Fantini, Opt. Expr. 6, 49 (2000).

4. G. Strangman, D. A. Boas and J. P. Sutton, Biol. Psychiatry 52, 679 (2002).

5. F.E.W Schmidt, M.E. Fry, E.M.C. Hillman, J.C. Hebden and D.T. Delpy, Rev. Sci. Instrum. 71, 256 (2000).

6. G. Gratton and M. Fabiani, Trends Cogn. Sci. 5, 357 (2001).

7. G. Maret and P.E. Wolf, Z. Phys. B 65, 409 (1987).

8. D. J. Pine, D. A. Weitz, J.X. Zhu and E. Herbolzheimer, J. Phys. France 51, 2101 (1990).

9. M. Heckmeier and G. Maret, Europhys. Lett. 34, 257 (1996).

10. D.A. Boas, L.E. Campbell and A.G. Yodh, Phys. Rev. Lett. 75,1855-1858 (1995).

11. C. Cheung, J.P. Culver, K.Takahashi, J.H. Greenberg and A.D. Yodh, Phys. Med. Biol. 46, 2053 (2001).

12. T. Durduran, G. Yu, M.G. Burnett, J.A. Detre, J.H., Greenberg, J. Wang, C. Zhou and A.G. Yodh, Opt. Lett. 29, 1766 (2004).

13. J. Li, G. Dietsche, S. E. Skipetrov, G. Maret, B. Rockstroh, T. Elbert and T. Gisler, J. Biomed. Opt. (2005, in press).

14. H. Ito, K. Takahashi, J. Hatazawa, S. G. Kim and I. Kanno, J. Cerebr. Blood Flow Metab. 21, 608 (2001). 